\title{
Numerical and analytical investigation towards performance enhancement of a newly developed rockfall protective cable-net structure
}

\author{
S. Dhakal ${ }^{1,3}$, N. P. Bhandary ${ }^{2}$, R. Yatabe $^{2}$, and N. Kinoshita ${ }^{2}$ \\ ${ }^{1}$ Graduate School of Science and Engineering, Ehime University, Matsuyama, Japan \\ ${ }^{2}$ Department of Civil and Environmental Engineering, Ehime University, Matsuyama, Japan \\ ${ }^{3}$ Nepal Engineering College, Pokhara University, Bhaktapur, Nepal \\ Correspondence to: S. Dhakal (shankeronly@gmail.com, shankerd@nec.edu.np)
}

Received: 15 August 2011 - Revised: 5 December 2011 - Accepted: 20 March 2012 - Published: 20 April 2012

\begin{abstract}
In a previous companion paper, we presented a three-tier modelling of a particular type of rockfall protective cable-net structure (barrier), developed newly in Japan. Therein, we developed a three-dimensional, Finite Element based, nonlinear numerical model having been calibrated/back-calculated and verified with the element- and structure-level physical tests. Moreover, using a very simple, lumped-mass, single-degree-of-freedom, equivalently linear analytical model, a global-displacement-predictive correlation was devised by modifying the basic equation - obtained by combining the principles of conservation of linear momentum and energy - based on the back-analysis of the tests on the numerical model. In this paper, we use the developed models to explore the performance enhancement potential of the structure in terms of (a) the control of global displacement - possibly the major performance criterion for the proposed structure owing to a narrow space available in the targeted site, and (b) the increase in energy dissipation by the existing U-bolt-type Friction-brake Devices - which are identified to have performed weakly when integrated into the structure. A set of parametric investigations have revealed correlations to achieve the first objective in terms of the structure's mass, particularly by manipulating the wire-net's characteristics, and has additionally disclosed the effects of the impacting-block's parameters. Towards achieving the second objective, another set of parametric investigations have led to a proposal of a few innovative improvements in the constitutive behaviour (model) of the studied brake device
\end{abstract}

(dissipator), in addition to an important recommendation of careful handling of the device based on the identified potential flaw.

\section{Introduction}

\subsection{A brief review of the companion paper}

In the companion paper Dhakal et al. (2011a), we introduced a particular type of rockfall protective cable-net structure (barrier), called Long-span Pocket-type Rock-net (abbreviated by LPR), developed newly in Japan, and discussed its modelling in three tiers. The expensive full-scale experimental modelling (full-scale test: FST) was followed by the cheaper and a bit simpler numerical and analytical modelling. For the time being, a configuration having $15 \mathrm{~m}$ span and $150 \mathrm{~kJ}$ designated impact energy capacity was taken as the reference structure (abbreviated by R-LPR). The geometry and composition of the structural model of the R-LPR is shown in Fig. 1. An experimentally calibrated and verified, Finite Element (FE) based, three-dimensional, nonlinear numerical model as well as a numerically calibrated (backcalculated), energy-momentum based, lumped-mass singledegree-of-freedom (SDOF), equivalently linearly elastic analytical model - capable of predicting the global-displacement of the structure - were successfully developed. Figure 2 shows the developed numerical and analytical models. The details of the model development are not reported in this paper for brevity. Nonetheless, to recapitulate for a quicker reference, a cylindrical block (length to diameter ratio of 2) 


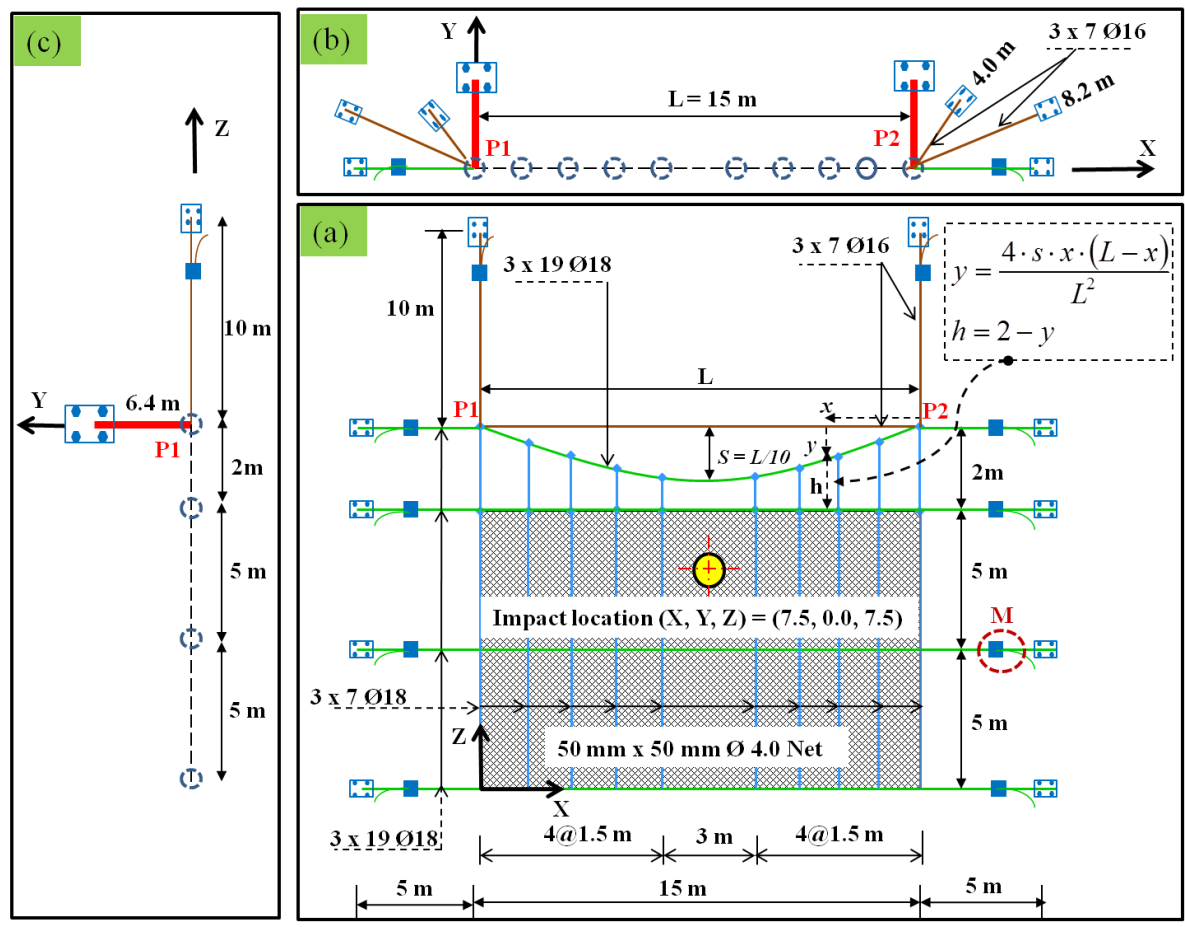

Fig. 1. The geometry and composition of the structural model of the R-LPR consisting of the net-cables-dissipators system as major and the idealized/generalized orientation of the support system as minor. (a) Front elevation. (b) Top view. (c) Side elevation viewed from the left towards the end-post P1. The maximum cable tension and the corresponding maximum slip-displacement were recorded at the the location $M$ having been highlighted. See Dhakal et al. (2011a) for more information.

of 1 ton mass was released from a certain level of the test set up profiled onto a specially prepared site (see Fig. 3) such that the block impacted the wire-net with a velocity of $17.33 \mathrm{~m} \mathrm{~s}^{-1}$ at a specified point located at the mid-span, three-forth height from the bottom edge of the wire-net. This resulted in the specified impact energy of $150 \mathrm{~kJ}$.

The system performed well; no damage was recorded and it was confirmed that the instrumented forces developed in the cables were well below the safety margin defined by the (yet conservative) Allowable Stress Design philosophy (see Dhakal et al., 2011a for further details). Moreover, given the very important condition that the targeted sites of LPR application typically have very limited space between the hillslope and the road-carriageway the former verification implied that the global out-of-plane displacement response of the wire-net (which may be simply referred to as "displacement" hereinafter) might be more vital in evaluating the performance of the structure. This response quantity which may appear to be a serviceability criterion (for the structure itself) is, in fact, an indirect measure of safety. It is in the sense that the larger displacement (say, above $3.5 \mathrm{~m}$ for a $150-\mathrm{kJ}$ rockfall event when the topography has recommended the set back of only $3.5 \mathrm{~m}$ ) is detrimental to the road traffic as the system may hit the latter although with reduced energy while restraining the falling rock-boulder.

\subsection{Research problem statement and objectives}

In the companion paper, although the usefulness of the proposed convenient forms of numerical and analytical (displacement predictive) models, for the various subsequent parametric analyses, was introduced in a few couples of sentences, the detailed investigation could not be covered. In fact, the proposed modelling, particularly the numerical one, might be utilized to investigate a set of yet effective and efficient configurations of LPR structures (including the use of a higher grade material) and the development of the respective performance correlations (design charts). Similarly, the models can be applied to investigate the effects of the various idealizations/assumptions on rockfall impact loading onto the structure (e.g. see Dhakal et al., 2011b, 2012). However, in the present paper, we do not change the geometrical framework of the studied R-LPR structure, assuming the existing structural form to be sufficiently new and innovative for the given constraint. Instead, we focus on utilizing the models to investigate how the system performance could be enhanced by changing the characteristic-parameters of the components in the existing framework.

The first issue of the performance enhancement is how to control the displacement response in an LPR structure, which ensures the safety of the protected zone as emphasized in Sect. 1.1; by keeping the stresses (particularly the 

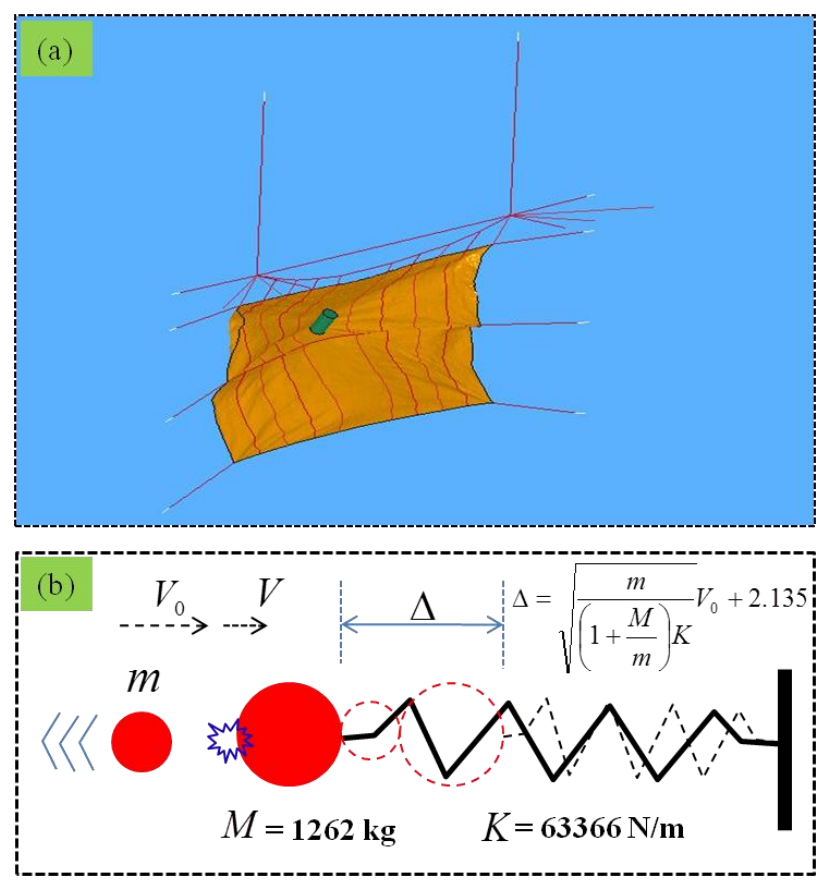

Fig. 2. The FE based nonlinear numerical model (a) and the Energy-Momentum based equivalently linear SDOF analytical model with the respective displacement-predictive correlation (b) of the R-LPR structure, in deformed states. Due to the fine discretization of the wire-net-equivalent shells in $50 \mathrm{~mm} \times 50 \mathrm{~mm}$ grid corresponding to the woven mesh size in reality, the individual finite elements are not seen in the FE model. It is noted that a mesh optimization study and/or a coupled modelling in the future may save some computational cost. See also Fig. 5.

forces in the cables since the net is purposefully made much stronger) within a safe limit. Figure 4 is shown to highlight the situation again. The second issue to be investigated is about achieving a greater energy absorption by the employed U-bolt-type Friction-brake (energy dissipating) Devices (UFDs).

The second issue emerges from a simple observation in the FST that the maximum slip-displacement reached only to some one-tenth of a meter when the maximum tension (maximum of all cables, at the location $M$ as highlighted in Fig. 1) reached as high as $90 \mathrm{kN}$. This is, in fact, against the general expectation of a larger value of maximum slip-displacement, say 0.5 to $1 \mathrm{~m}$ or even more; for example, as prevails in the contemporary ring-type brake devices commonly used in the flexible barrier systems developed in Europe (e.g. see Cazzani et al., 2002; Volkwein, 2004), or as also revealed from a study inside Japan (see Tajima et al., 2009), or as observed in one of the cases of a $30-\mathrm{m}$ span (400-kJ capacity) LPR itself in the past test verification campaign (see Kinoshita, 2009). Having said that, it is to be noted that the referenced absolute values of slip-displacements only may not be taken as the sole evaluation criteria of energy dissipation. The

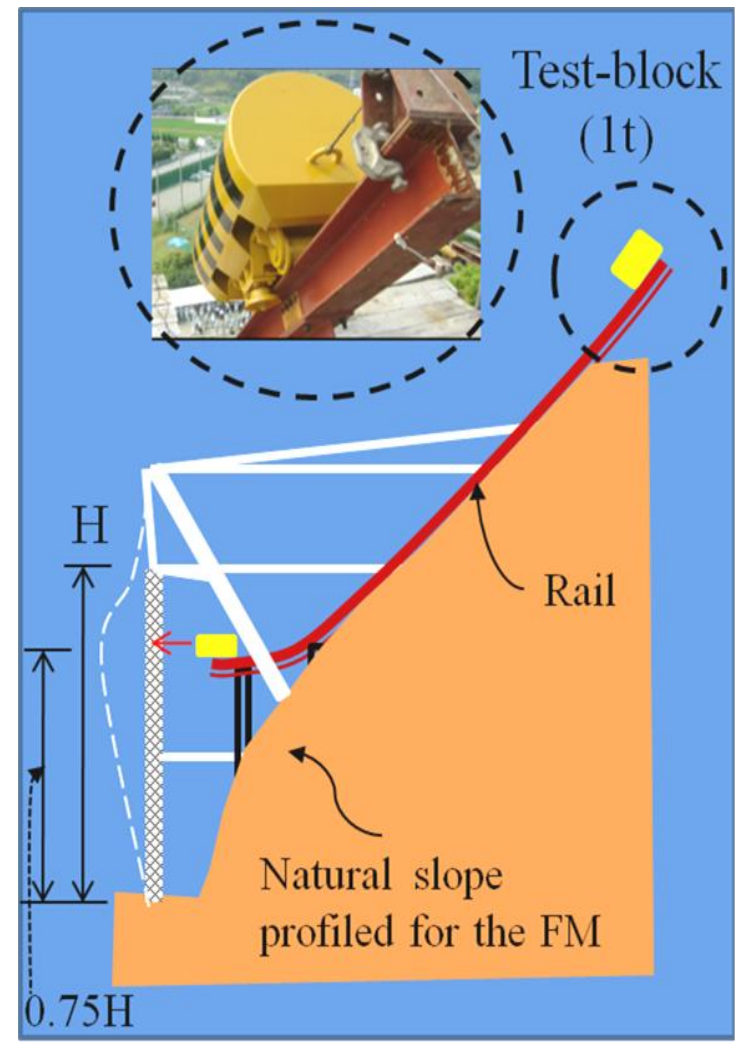

Fig. 3. The FST scheme of the R-LPR structure.

corresponding tension in the cables being installed with the brake devices (dissipators) should also be taken into account. Or, more reliably, the proportion $\left(E_{d}\right)$ of total impact energy $\left(E_{0}\right)$ - the incident kinetic energy of the impacting block dissipated by the devices should act as the most effective measurement of their effectiveness when integrated into the structural system. We may hereinafter refer the measurement to as the "energy dissipation efficiency" of the brake devices. In such a case, the minimum expectation may be generally taken above one-third (e.g. see Tajima et al., 2009). However, as may be read from the energy transformation time history numerically evaluated in this work as detailed out in Sect. 3.1, the large number of the UFDs integrated into the structure dissipated much less. It is only about $15 \%$ of the total impact energy to be fully resisted by the structure while undergoing the maximum displacement. Thus, this revealed the need of an investigation to enhance the impact energy resistance performance of the R-LPR structure by identifying the potential weaknesses of the integrated brake devices and the possible solution/s to improve them (Dhakal et al., 2011c).

Thus, enhancing the structural behaviour of the newly developed system of LPR (again taking the R-LPR as a reference case) by (a) controlling (minimizing) the displacement demand and (b) improving the brake technology (i.e. the energy dissipation efficiency) are the two major original 


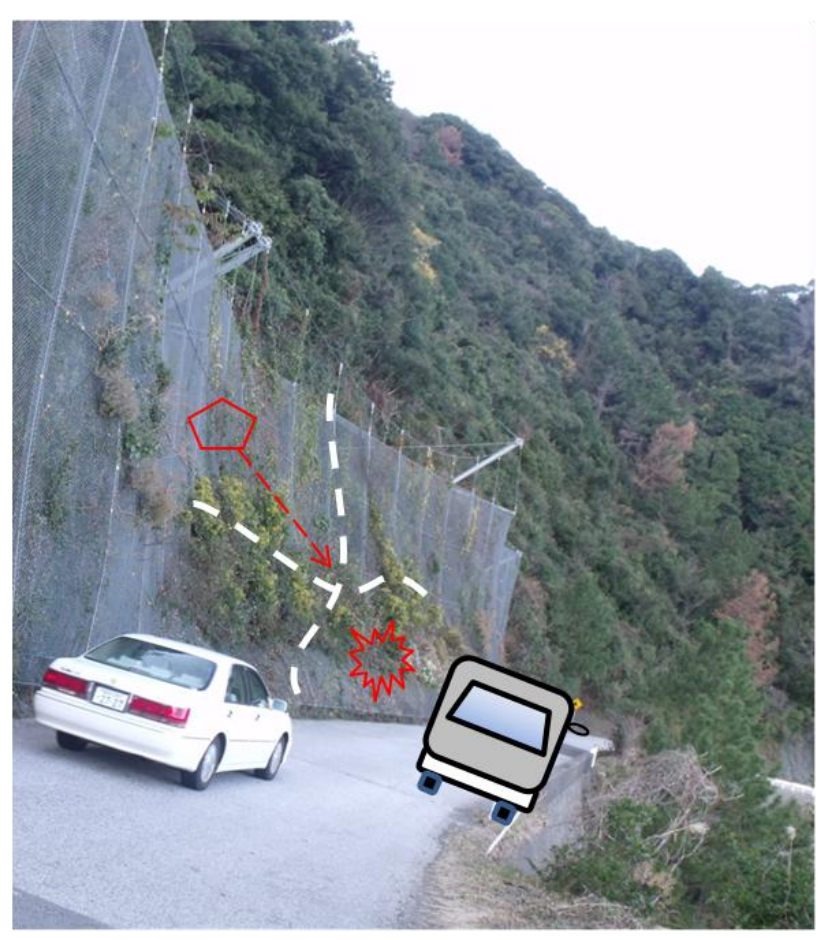

Fig. 4. A simple schematic illustration of the reason for putting the (out-of-plane) displacement response into priority in evaluating the performance of an LPR structure. Actually, the structure is constructed very close to the road-carriageway and the risk onto the road traffic due to a larger displacement of wire-net needs to be checked. Illustrations are drawn over a recent photo of a wellfunctioning structure from a site along Kurei-Suzaki Line road in Kochi Prefecture of Japan, installed later 2009.

objectives of this paper. Here, the beauty of the convenient methods of numerical and analytical modelling as provoked in the companion paper is automatically illustrated. Again, despite being specific to a design of LPR, the approach, methodologies, results and interpretations contained in this work may be useful to enhance the understanding of other similar types of protective system.

\section{Towards controlling the displacement}

\subsection{Idea and methodology}

The consideration of structural mass in addition to the usually practiced method by cushion material for the protection against impact actions is emerging (e.g. Lam et al., 2010; Yang et al., 2011; Dhakal et al., 2011a; Ali et al., 2011). As such, the wire-net is the major structural component of the rockfall restraining LPR system and contributes more than $70 \%$ to the total mass. Therefore, it is the mass of the wirenet which should be manipulated to achieve effective displacement control and enhance system performance.

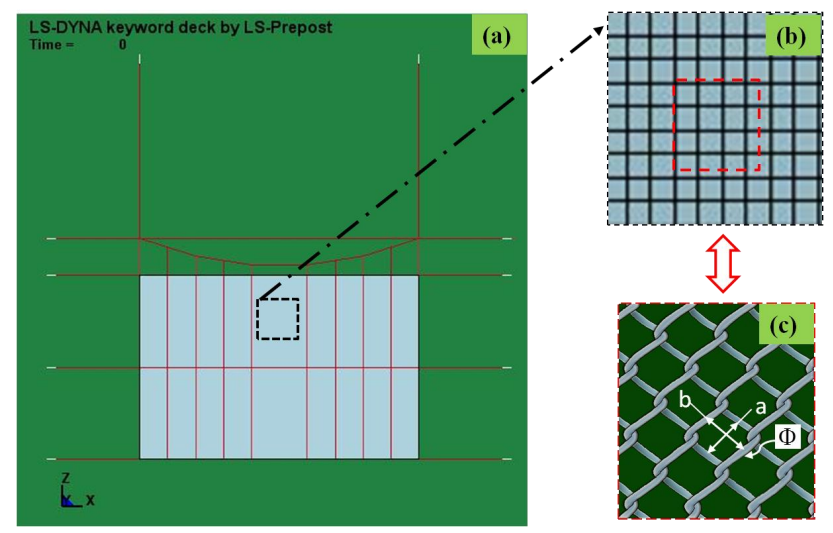

Fig. 5. The wire-net in an LPR system. (a) Numerical model of the R-LPR, where wire-net is modelled equivalently by continuum (shell) finite elements; due to very dense grid of $50 \mathrm{~mm} \times 50 \mathrm{~mm}$, the discretization is not seen. (b) Zoomed view of the shell elementmesh cropped from the former model. (c) Schematic drawing of the woven wire-net (mesh) in reality; where the mass per unit area, for given mesh size such as $(a \times b)$ and material, are specified as per the diameter $(\Phi)$ of the constituting wires; e.g. $4.6 \mathrm{~kg} \mathrm{~m}^{-2}$ for $50 \times 50 \times 4 \mathrm{~mm} \Phi$, and $7.1 \mathrm{~kg} \mathrm{~m}^{-2}$ for $50 \times 50 \times 5 \mathrm{~mm} \Phi$.

Figure 5 highlights the wire-net component in an LPR structure by referring to the numerical model of the R-LPR, and shown together is the woven mesh in reality. Now, as per the mass of the wire-net (or wire-mesh), it is normally specified in terms of the "mass per unit area". For example, the specified values for the $50 \mathrm{~mm} \times 50 \mathrm{~mm} \times 4 \mathrm{~mm} \Phi$ and $50 \mathrm{~mm} \times 50 \mathrm{~mm} \times 5 \mathrm{~mm} \Phi$ wire-nets (the former dimensions in the form of $\mathrm{a} \times \mathrm{b}$ being the size of the grid in the mesh and $\Phi$ the diameter of the weaving wire) are $4.6 \mathrm{~kg} \mathrm{~m}^{-2}$ and $7.1 \mathrm{~kg} \mathrm{~m}^{-2}$, respectively. The latter is about 1.5 times heavier than the former. The real wire-net has material-less holes in their grids. However, in the verified numerical modelling of the R-LPR, exercised in the code of LS-DYNA as described in the companion work, we conveniently modelled the $50 \mathrm{~mm} \times 50 \mathrm{~mm} \times 4 \mathrm{~mm} \Phi$ mesh with the equivalent continuum shell elements by utilizing the BelytschkoTsay Shell formulation and Fabric material model available in the code (see Dhakal et al., 2011a for more information). The equivalent shell elements were assumed to have the density equal to that of ordinary steel $\left(7840 \mathrm{~kg} \mathrm{~m}^{-3}\right)$ and hence the equivalent thickness equalled to $0.587 \mathrm{~mm}$ (4.6 divided by 7840 ).

In the shell-equivalent modelling, the equivalent mass of the wire-net may be expressed in terms of either density or thickness. Absolutely speaking, the mass of the wire-net $(M)$ may be increased by proportionately increasing its density $(\rho)$ (i.e. keeping the thickness $(t)$ constant as the area $(A)$ is automatically constant when we are confined to a particular design such as the R-LPR). Alternatively, the thickness can be increased in proportion to the desired increment, say by $(n)$, in the mass by keeping the density constant; as shown 


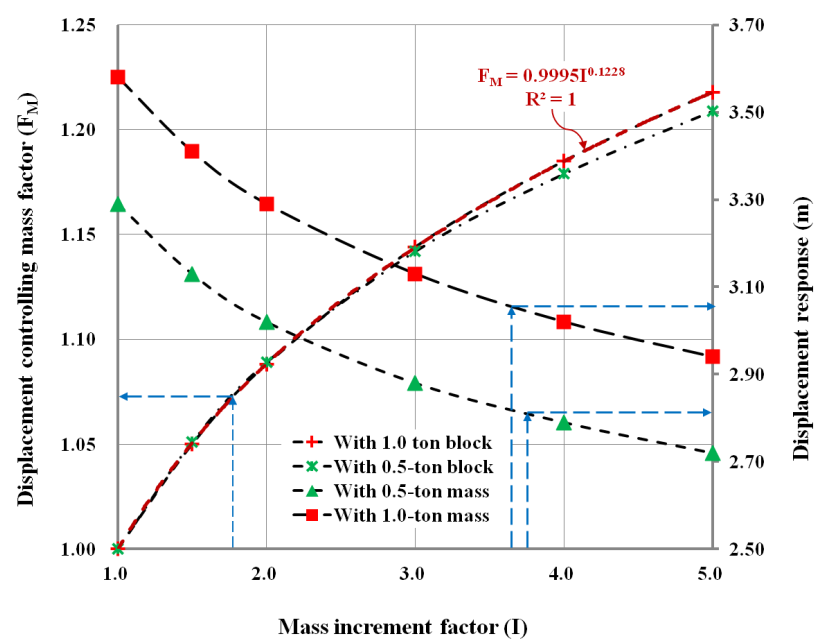

Fig. 6. Displacement controlling mass factors for two sets of impact conditions of the R-LPR structure resulting in the same specified impact energy of $150 \mathrm{~kJ}$. The first is with 1.0-ton block and the second with 0.5 -ton block. The shown correlation equation is with the 1.0-ton mass.

by a simple formula in Eq. (1). However, when the mass is achieved via thickness, the stiffness property also increases. By subtracting the displacement response by the density increment from that by the thickness increment, we may visualize the contribution by stiffness only

$M=\rho \cdot A \cdot t \Rightarrow n \cdot M=(n \cdot \rho) \cdot A \cdot t=\rho \cdot A \cdot(n \cdot t)$.

Towards quantifying the control of displacement by the mentioned idea, we utilize both the analytical and numerical models. The generalized form of the displacement predictive correlation (as already shown in Fig. 2) is also reproduced in Eq. (2). In the equation, $(m)$ and $\left(V_{0}\right)$ are respectively the mass and velocity of the impacting block and $(M)$ and $(K)$ are respectively the equivalent mass and the equivalent stiffness of (the kinematically equivalent), SDOF model. Here, the units are: $(m)$ in $\mathrm{kg},\left(V_{0}\right)$ in $\mathrm{m} \mathrm{s}^{-1}$ and the resulting displacement $(\Delta)$ in $\mathrm{m}$

$\Delta=\sqrt{\frac{m}{\left(1+\frac{M}{m}\right) K}} V_{0}+2.135$

Specifically, for the studied R-LPR structure of the geometry and composition as shown in Fig. 1, the numerically calibrated (back-calculated) values of the equivalent mass and equivalent stiffness were found to be $1262 \mathrm{~kg}$ and $63366 \mathrm{~N} \mathrm{~m}^{-1}$, respectively (see Dhakal et al. (2011a) for more information). It is noted that, in order to assess the effects of the parameters such as the density and the thickness of the equivalent shells, we couldn't use the analytical model and had to simulate through the numerical model. The details of the relevant parametric computations are explained in Sect. 2.2.

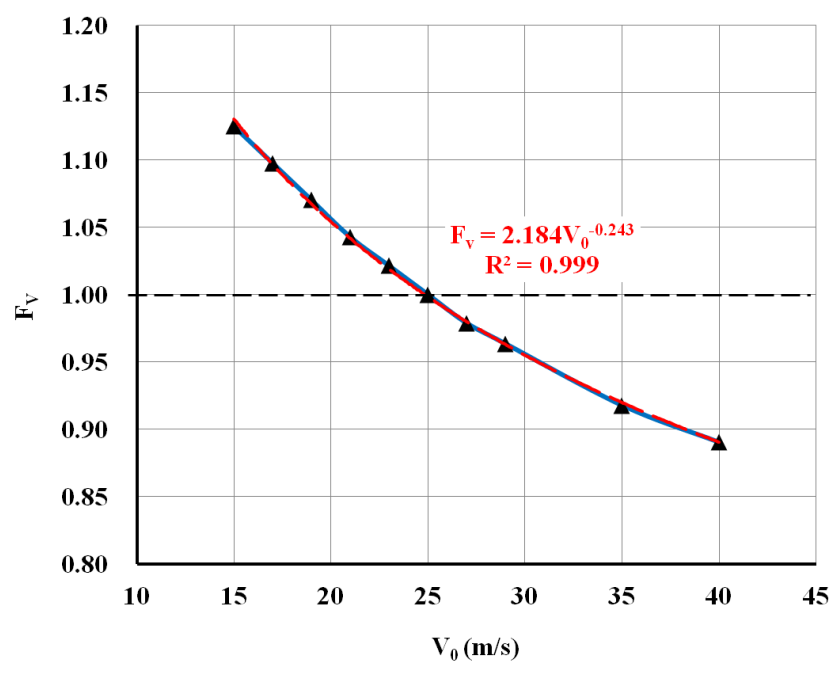

Fig. 7. The "Displacement response velocity factors" for the R-LPR structure at $150 \mathrm{~kJ}$.

\subsection{Results and discussion}

Using Eq. (2), we first predicted the maximum displacement response $\Delta$ (or D) when the equivalent mass of the structure $(\mathrm{M})$ is increased by the factors of $1.5,2,3$, etc. (we define them as the "Mass increment factor" (I), which as described earlier, shall be achieved primarily via wire-net. We define the quantity obtained by normalizing the displacement response at a certain mass increment factor (I) by the original displacement response (i.e. at $\mathrm{I}=1$ ) as the "Displacement controlling mass factor" $\left(F_{\mathrm{M}}\right)$ or $\left(F_{\mathrm{M}}(\mathrm{I})\right)$, as presented mathematically in Eq. (3)

$F_{\mathrm{M}}(\mathrm{I})=\frac{\mathrm{D}(\mathrm{I}=\mathrm{I})}{\mathrm{D}(\mathrm{I}=1)}$.

The respective results are presented accordingly in Table 1 and Fig. 6. The impact scenario equivalent to the FST (i.e. the block of mass of 1 ton impacting the structure with the velocity of $17.33 \mathrm{~m} \mathrm{~s}^{-1}$, thereby resulting the specified impact energy of $150 \mathrm{~kJ}$ ) was assumed. The structure's equivalent stiffness was kept unchanged at $63366 \mathrm{~N} \mathrm{~m}^{-1}$.

A similar set of displacement responses were determined from numerical simulations: first by varying the density of wire-net while keeping the thickness constant, and then varying the thickness while keeping the density constant. We define the corresponding displacement controlling factors obtained by normalizing the displacement responses; with respect to the original value as the "Displacement controlling density factor" $\left(F_{\mathrm{d}}\right)$ and the "Displacement controlling thickness factor" $\left(F_{\mathrm{t}}\right)$. The results are presented accordingly in Table 2.

For the purpose of analysis, using Eq. (2) we derived a set of displacement response data for the structure for the various combinations of velocity $\left(\mathrm{V}_{0}\right)$ and the corresponding mass $(\mathrm{m})$ of the block within a practical range, yielding 
Table 1. Analytical computation of the Displacement controlling mass factors for the R-LPR structure at two different impact scenarios each resulting in the same specified impact energy of $150 \mathrm{~kJ}$. I = Mass increment factor; $\mathrm{D}=$ displacement response; $\mathrm{M}=$ structural mass; $F_{\mathrm{M}}=$ Displacement controlling mass factor.

\begin{tabular}{llllll}
\hline \multirow{2}{*}{$\mathrm{I}$} & $\mathrm{M}(\mathrm{kg})$ & \multicolumn{3}{c}{$m=1.0 \mathrm{t}$} & \multicolumn{2}{c}{$m=0.5 \mathrm{t}$} \\
\cline { 3 - 6 } & & $\mathrm{D}(\mathrm{m})$ & $F_{\mathrm{M}}$ & $\mathrm{D}(\mathrm{m})$ & $F_{\mathrm{M}}$ \\
\hline 1.0 & 1262 & 3.58 & 1.000 & 3.29 & 1.000 \\
1.5 & 1893 & 3.41 & 1.050 & 3.13 & 1.051 \\
2.0 & 2524 & 3.29 & 1.088 & 3.02 & 1.089 \\
3.0 & 3786 & 3.13 & 1.144 & 2.88 & 1.142 \\
4.0 & 5048 & 3.02 & 1.185 & 2.79 & 1.179 \\
5.0 & 6310 & 2.94 & 1.218 & 2.72 & 1.209 \\
\hline
\end{tabular}

Table 2. Numerically computed Displacement controlling density factors and Displacement controlling thickness factors for the RLPR structure at the FST loading scenario. I= Mass increment factor; $\mathrm{D}=$ Displacement response, $F_{\mathrm{d}}=$ Displacement controlling density factor; $F_{\mathrm{t}}=$ Displacement controlling thickness factor.

\begin{tabular}{llllll}
\hline \multirow{2}{*}{$\mathrm{I}$} & \multicolumn{2}{c}{ Via density } & \multicolumn{2}{c}{ Via thickness } & \multirow{2}{*}{$F_{\mathrm{t}}-\mathrm{F}_{\mathrm{d}}$} \\
\cline { 2 - 5 } & $\mathrm{D}(\mathrm{m})$ & $F_{\mathrm{d}}$ & $\mathrm{D}(\mathrm{m})$ & $F_{\mathrm{t}}$ & \\
\hline 1.0 & 3.60 & 1.000 & 3.60 & 1.000 & 0.000 \\
1.5 & 3.38 & 1.065 & 3.16 & 1.139 & 0.000 \\
2.0 & 3.16 & 1.139 & 3.00 & 1.200 & 0.061 \\
3.0 & 2.88 & 1.250 & 2.77 & 1.300 & 0.050 \\
\hline
\end{tabular}
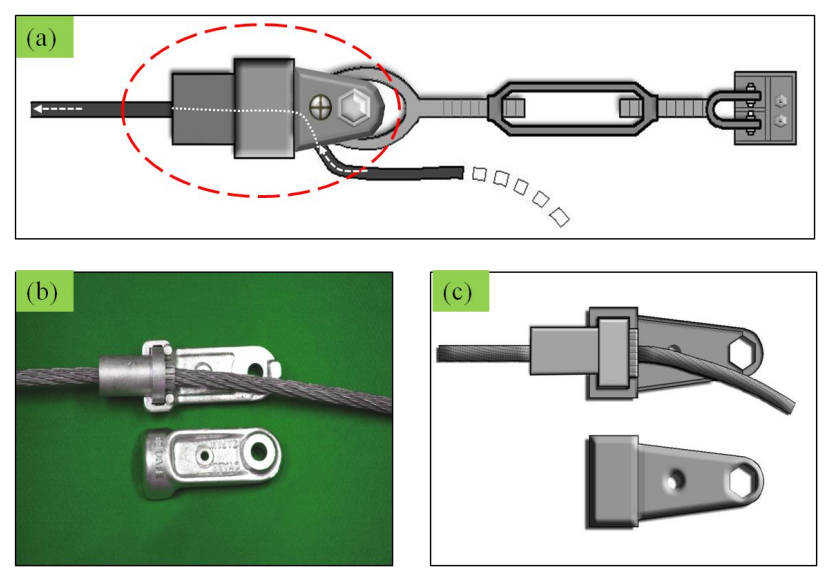

Fig. 8. The UFD device used in the LPR structures. (a) Schematic diagram of the device integrated with cable and finally anchored at the end. (b) A photo of the disassembled device. (c) Schematic drawing of the disassembled device.

the same specified impact energy of $150 \mathrm{~kJ}$ (kinetic energy equals half the product of mass and velocity squared) whilst keeping the structural properties the constant of the R-LPR. Here, we normalized the displacement response at any velocity $\left(\mathrm{V}_{0}\right)$ with respect to that due to the threshold impact velocity of $25 \mathrm{~m} \mathrm{~s}^{-1}$ (i.e. $\mathrm{V}_{0}=25 \mathrm{~m} \mathrm{~s}^{-1}$ ) recommended in the European code, ETAG027 (see Peila and Ronco, 2009). We define the corresponding dimensionless response quantity as the "Displacement response velocity factor", $\left(F_{\mathrm{V}}\right)$ or $\left(F_{\mathrm{V}}\left(\mathrm{V}_{0}\right)\right)$, as shown mathematically in Eq. (4)

$$
F_{\mathrm{V}}\left(\mathrm{V}_{0}\right)=\frac{D\left(\mathrm{~V}_{0}=\mathrm{V}_{0}\right)}{D\left(\mathrm{~V}_{0}=25\right)} .
$$

The results are presented accordingly in Fig. 7.

Furthermore, because the analytical model is very easy to handle, we became interested to see also how the displacement would vary with the changing structural mass (i.e. the Mass increment factors (I) of 1.5, 2, 3, etc.), if the hazard situation was such that the designated impact energy of $150 \mathrm{~kJ}$ resulted due to a smaller mass (say, 0.5 ton, the size that frequently occurs in Japanese hills according to a study by Matsuo et al., 1999); falling from a greater height or impacting with a higher incident velocity $\left(24.5 \mathrm{~m} \mathrm{~s}^{-1}\right.$ for the 0.5 -ton block). The results are again presented in Table 1 and Fig. 6 for better comparison.

Now let's interpret and discuss the obtained results in brief.

The results in Table 1/Fig. 6 reveal interesting correlation of how the desired control of displacement may be achieved via the structural mass. In particular, a picture of achieving the control of displacement in an LPR structure through the increment in the mass of the wire-net, in terms of its density and equivalent thickness, can be depicted from Table 2 . The considered parameters of the wire-net in reality may be improved through the spacing of the woven mesh and/or the diameter of the weaving wire.

Referring to Fig. 6, a closely fitting power correlation between " $F_{\mathrm{M}}$ " and "I" in the form of Eq. (5) has been obtained.

$F_{\mathrm{M}}=a \mathbf{I}^{b}$.

The coefficients $(a)$ and $(b)$ may be termed as the "Displacement controlling mass coefficients" or more precisely the "RLPR displacement controlling mass coefficients". The values of the coefficients for a specific case of the impact equivalent 

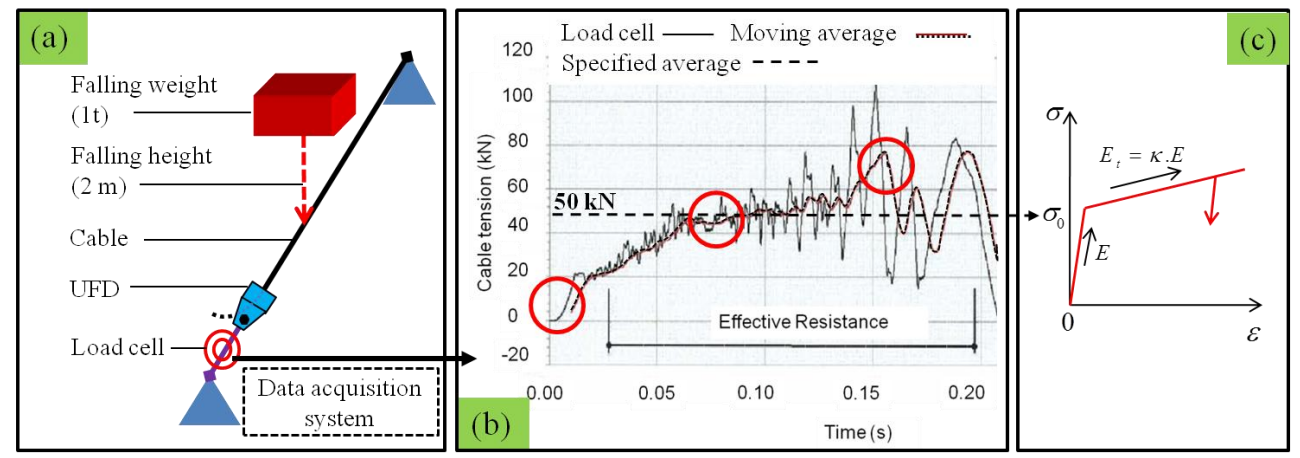

Fig. 9. Constitutive modelling of the UFDs modelled by the equivalent Truss element in the developed numerical (FE) model of the R-LPR. (a) Scheme of the falling-weight impact test (FWT) conducted by Besafe (2006). (b) A typical time history of the tension force recorded in the FWT, which very precisely recommended the average slip-tension designation of $50 \mathrm{kN}$. (c) Elastic-linearly plastic/hardening constitutive model (Plastic Kinematic in LS-DYNA) considered in the FE modelling. Reproduced from Dhakal et al. (2011a).

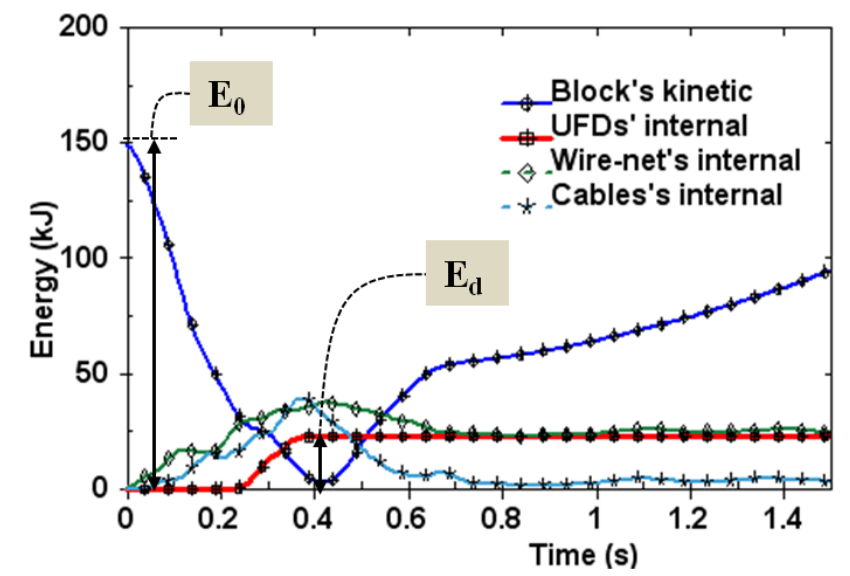

Fig. 10. Numerically simulated time history highlighting the energy dissipation by the UFDs in the the R-LPR in the FST, where the block carrying $150 \mathrm{~kJ}$ of impact energy hit the structure at the predefined point in the wire-net.

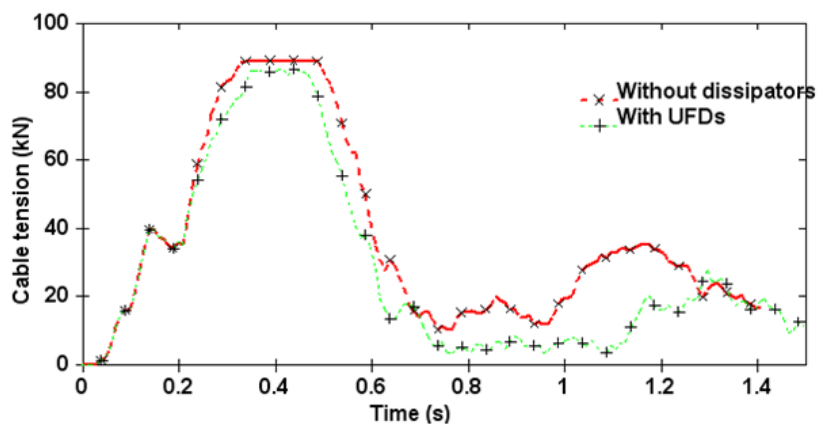

Fig. 11. Numerically simulated evolution of maximum tension in the cable (at location $M$ in Fig. 1) with and without UFDs integrated into the system, subjected to the same impact energy of $150 \mathrm{~kJ}$.

to the FST verification of the R-LPR have been determined analytically to be approximately 0.999 and 0.123 .
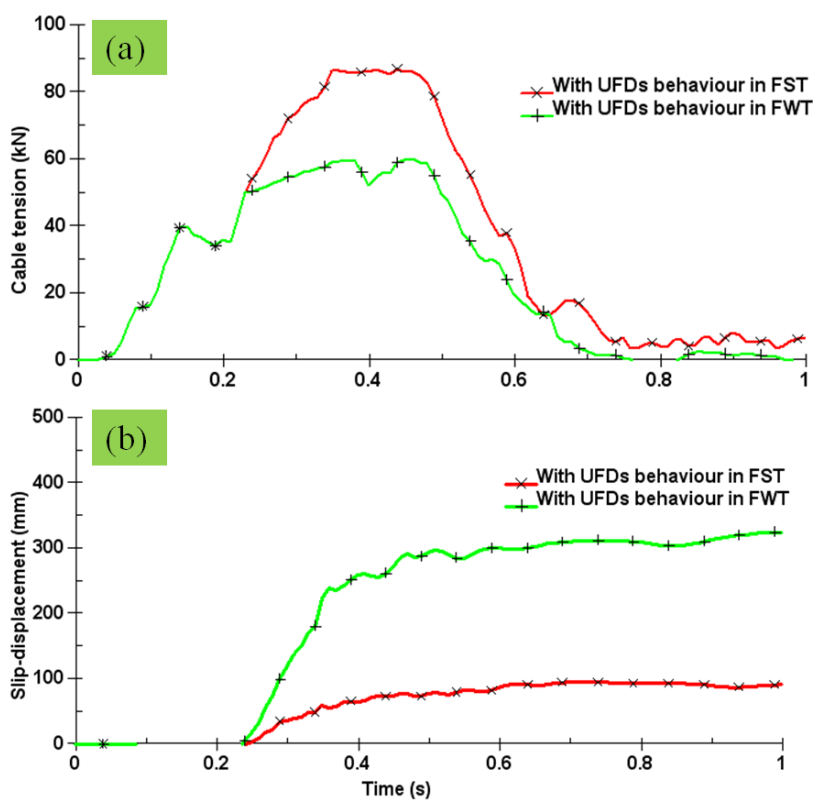

Fig. 12. Numerically simulated evolution of maximum cable tension (a) and slip displacement (b) responses of the R-LPR structure when integrated with the UFDs having their constitutive behaviour as exhibited in structure-level impact test (FST) and element-level (isolated) test of the UFDs (FWT).

Moreover, referring to the relatively smaller differences between the values of displacement controlling density factor and displacement controlling thickness factor, while both are contributing to the equal values of mass increment factor as shown in Table 2, it is seen that the mass property of the structure has played a more significant role in controlling the displacement than the stiffness property.

To materialize the control of displacement through the higher value of " $F_{\mathrm{M}}$ ", in addition to enhancing the mass of the wire-net such as by increasing the spacing of grids or the 


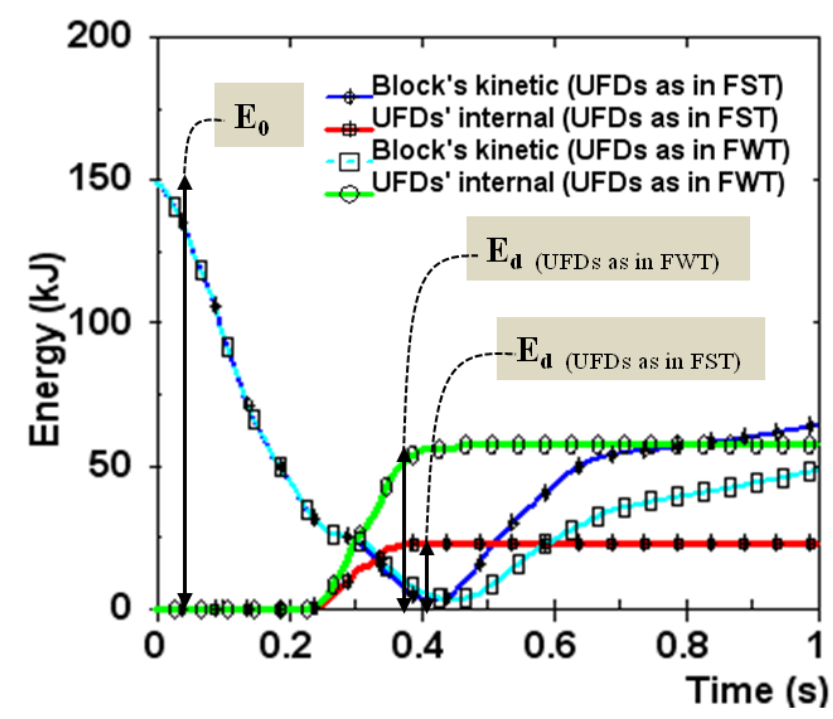

Fig. 13. Comparison of numerically simulated energy transfomation time histories and energy dissipation by the UFDs having different constitutive behaviours when integrated into the R-LPR structure. The red (dotted) lines correspond to the UFD behaviour exhibited in the FST (with the computed hardening parameter equal to 0.001) whilst the blue (solid) lines correspond to that in the FWT (with the computed hardening parameter of 0.0001 ).

diameter of the weaving wires, it may be recommended that the larger value of "I" may also be achieved alternatively; by going for a hybrid structure, for example, consisting of some double-layer mesh. A separate investigation, however, deems necessary to evaluate the effectiveness of such a recommendation.

Additionally, although it may not be exactly suitable to our objective of controlling the displacement, the effects of the block velocity or mass (or size at a given material) in the displacement performance of an LPR structure have been very clearly observed. Referring to Fig. 7, another closely fitting power law (now with negative exponent) between " $F_{\mathrm{V}}$ " and " $\mathrm{V}_{0}$ " in the form of Eq. (6) has been obtained

$F_{\mathrm{V}}=c \mathrm{~V}_{0}^{-d}$.

The coefficients $(c)$ and $(d)$ may be termed as the "Displacement response velocity coefficients" or more precisely the "R-LPR displacement response velocity coefficients". The values of the coefficients determined based on the analyses of the R-LPR are approximately equal to 2.184 and 0.243 , respectively. Interestingly, at velocities lower than $25 \mathrm{~m} \mathrm{~s}^{-1}$, such as the value employed in the FST campaign of LPRs, intuitively chosen, assuming that it might be the general case in the low-to-moderate rockfall hazard sites, $\left(F_{\mathrm{V}}\right)$ has an increasing trend. This consideration is thus safer when the global displacement response becomes typically important for a barrier like LPR. The results reveal that the LPR system is analogous to the single module ("single panel") cable
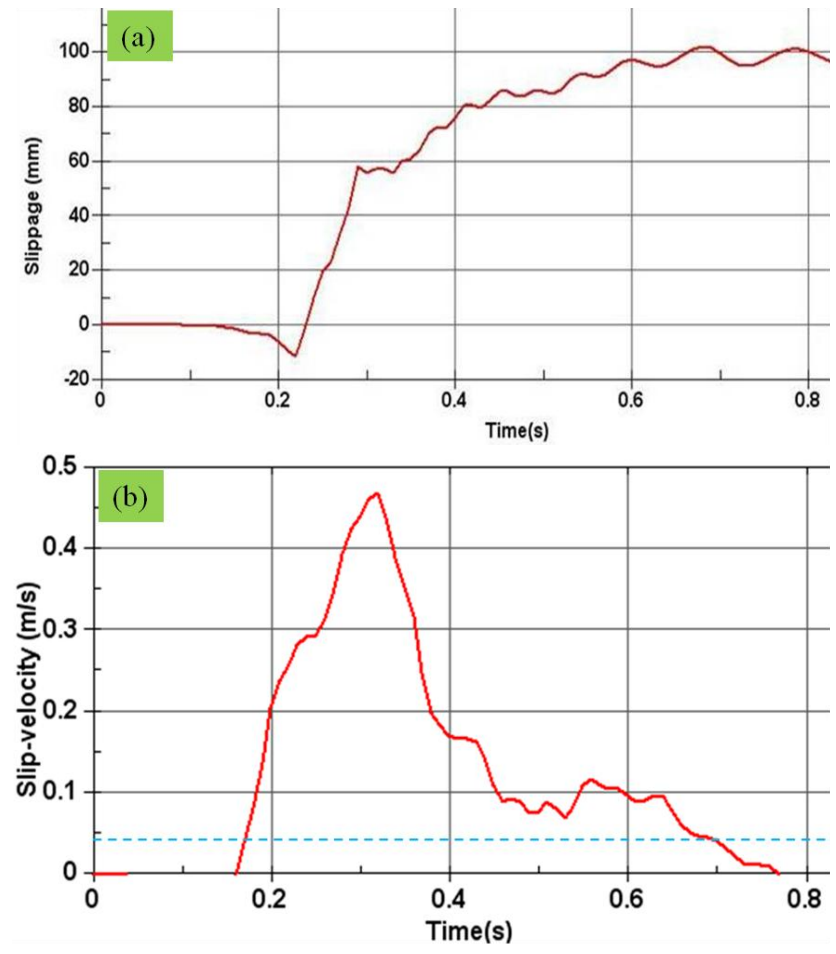

Fig. 14. Numerically simulated time histories of maximum slipdisplacement (slippage) of the UFD device (at location $M$ in Fig. 1) corresponding to the FST scenario of the R-LPR (a) and of the slip-velocity obtained by differentiating the slip-displacement w.r.t. time (b). The dotted (blue) horizontal line marks the assumed threshold slip-velocity of $0.04 \mathrm{~m} \mathrm{~s}^{-1}$ only below which the coefficient of dynamic frition varies significantly.

net system studied by Cazzani et al. (2002), which offered higher displacement response with bigger mass (or size) of the impacting block. This is convincing because the LPR design is relatively stiffer and is a one-panel structure, unlike the system of highly flexible barriers commonly practised in Europe (see Cantarelli et al., 2008). The smaller displacement by a larger block in a relatively stiffer structural system like the LPR may be interpreted in terms of the contact stiffness which can be better visualized with a lumped mass, twodegree-of-freedom (2DOF) model of the structure (see Lam et al., 2010 or Yang et al., 2011). The detailed analysis is left over assuming beyond the scope of this paper. Nonetheless, in brief, such a model is composed of the impacting block and the LPR structure as the two lumped masses; the former being connected to the latter with a spring of stiffness $\left(k_{1}\right)$ that is assumed to work based on the Hertz law, and the latter being connected to the support with another spring $(K)$ representing the structural stiffness as we have worked out in the SDOF model. Fundamentally, the smaller block causes larger local deformation and hence, the result is a lower value of the stiffness " $k_{1}$ " at the contact interface. This, in turn, causes the lower impact actions to be transferred into the 


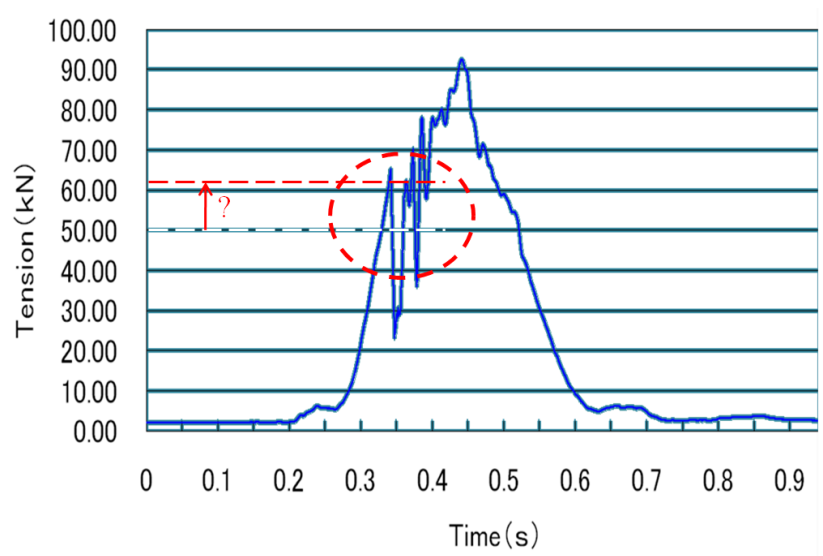

Fig. 15. The instrumented time history of maximum tension in cable (at $M$ in Fig. 1) in the FST of the R-LPR, in relation to the detection of the possible flaw under discussion. The dotted circle in the figure emphasizes the portion of the time history when the device must have slipped. The lower dotted horizontal line indicates the designated level of slip-tension (i.e. $50 \mathrm{kN}$ ) while the upper dotted horizontal line represents the level of actual slip-tension likely to have occured in the FST as inferred from the discontinuity in the history.

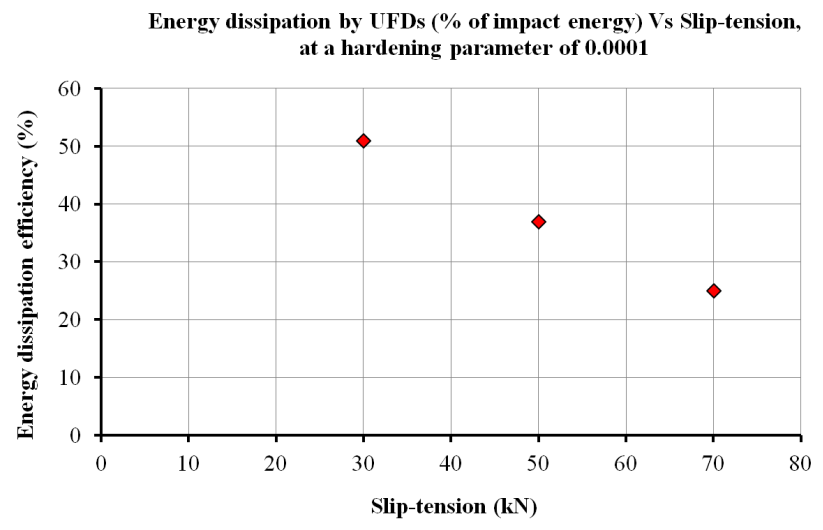

Fig. 16. Numerically simulated energy dissipation performance (efficiency) of the UFDs integrated into the R-LPR structure as affected by the slip-tension values at a given hardening parameter.

structure and hence, the result is relatively smaller global displacement of the structure. The situation is reversed with a larger size of the block. Volkwein et al. (2005) or Volkwein et al. (2009) describe the circumstance saying that: the loading of the impact area is higher for smaller and faster blocks compared to larger and slower ones with the same kinetic energy; however, the loading for the remaining structure is reduced.

Interestingly, referring to Fig. 7, we tend to outline another valid proposition for the LPR structures. From costbasis, flexible barriers and embankments are comparable (e.g. Yoshida, 1999) although the latter have higher energy capacity. The advantage of the LPR-like barriers against the embankments or rock-sheds, primarily in low-to-medium impact energy scenarios and in narrow-set-back sites, is obvious. Further to this, what seems to have been observed is that, even in the sites with wider set-backs, and where the hazard characteristics be such that the smaller size, higher velocity boulders are more dominating, the LPR systems (characterised with strong wire-net and smaller grids) are likely to be more effective as they exhibit better performance with smaller sized blocks. In fact, earthen embankments or geo-cells (e.g. Peila, 2011) may be most effective for such (smaller size, higher velocity) impacts. This phenomenon can again be described with the $2 \mathrm{DOF}$ modelling. In very short, an embankment (geo-cell) will effectively prolong the contact time between the falling boulder and the target and hence increase the natural period of the block-frontal spring system $\left(\mathrm{T}_{\mathrm{m}}\right)$. Yang et al. (2011) have developed a generic chart to predict the displacement demand $(\Delta)$ by a structure as the function of the ratio of the natural period of the blockfrontal spring system to that of the structure (i.e. $T_{\mathrm{m}} / T_{\mathrm{M}}$; which the authors define as the "period ratio"), together with the ratio of the mass of the structure to that of the impacting block (i.e. $\mathrm{M} / \mathrm{m}$; which the authors define as the "mass ratio") as another variable. Therein, a very clear picture of the significant decrease in the value of $(\Delta)$ with the increasing $\left(T_{\mathrm{m}} / T_{\mathrm{M}}\right)$ is shown and is particularly smaller for higher values of the mass ratio. Now, the smaller the boulder the higher is the value of the mass ratio. Therefore, favoured both by the period ratio and the mass ratio, the rockfall protective embankment structures are particularly effective for smaller boulders falling with high speed. Now, back to the comparison, if the capacity of the LPR-like structures increased appreciably by learning from the development of the highly flexible barriers used in Europe (which developed the capacity by some twenty times in approximately two decades!), they could even challenge the embankment systems. Encouragingly, as investigated in Sects. 3.2 and 3.3, there seems to be an ample opportunity to upgrade the existing lowercapacity designs of the LPR structures to higher designations simply by improving the constitutive behaviours of the UFDs.

\section{Towards increasing the energy dissipation efficiency of brakes}

\subsection{Low energy dissipation efficiency in existing system}

Figure 8 highlights the structure of the UFD device utilized in the newly developed structural system of LPR. In particular, such devices integrated with the studied structure of R-LPR had a designated slip-tension of $50 \mathrm{kN}$. In the FE numerical modelling (Dhakal et al., 2011a), the UFDs were modelled with Discrete Beam Truss elements of length $0.5 \mathrm{~m}$ and cross sectional area of $1000 \mathrm{~mm}^{2}$, having an elastic-linearly plastic/hardening constitutive model - the "Plastic Kinematic" 
material model available in LS-DYNA (LSTC, 2007). The model idealization was based on the observation of the behaviour of the devices in their element-level experiments (falling weight test: FWT). The process is summarized in Fig. 9. The modelled yield stress (physically, the slip-stress) of the equivalent truss elements, corresponding to the designated slip-tension of $50 \mathrm{kN}$, was computed to be $50 \mathrm{MPa}$. The initial elastic modulus was assumed equivalent to that of an ordinary steel bar $\left(2.1 \times 10^{11} \mathrm{~N} \mathrm{~m}^{-2}\right)$ connecting the U-head and the anchor. Referring to the performance of the devices in the FST (with the maximum observed slip-displacement of only some one-tenth of a meter while the corresponding cable-tension had reached as high as $90 \mathrm{kN}$ ), the hardening parameter $(k)$ of the adopted Plastic Kinematic constitutive model, to successfully simulate the FST behaviour, was computed to be 0.001 . It is noted, however, that the after-slip behaviour of the device was different in its FWT, giving the recorded slip-displacement of, as high as, $50 \mathrm{~cm}$ (Besafe, 2006), and this calculates the hardening parameter to be 0.0001 .

To elucidate the problem defined in Sect. 1.2, we refer to the numerically simulated time history, presented in Fig. 10, that highlights the energy dissipation by the UFDs (defined by the "UFDs' internal") when integrated into the R-LPR and subjected to an impact corresponding to the FST. The results were derived from the "History $>$ Material $>$ energies" option inside the postprocessor LS-PREPOST. The internal energies of the wire-net and cables are also shown in the figure as additional information. These components also develop kinetic energy during simulation while the UFDs' kinetic energy was noted negligible throughout the history. Anyway, clearly depicted from Fig. 10 is the unexpectedly low energy dissipation by the UFDs. The energy dissipation by such a large number of dissipators installed into the structure expressed in terms of the ratio $E_{d} / E_{0}$ - which we call as the "energy dissipation efficiency" - is only around $15 \%$. This value is less than even half of the average value obtained by Tajima et al. (2009), for instance. This is not encouraging and may not be taken as satisfactory, despite the very fact that the structure is usually expected to have to be capable of fully resisting the applied impact energy while undergoing maximum displacement (observed at around $0.4 \mathrm{~s}$ ), and the structure performed very well in that sense. It was declared mechanically quite safe and serviceable too, assuming that the set-back (clearance) available in the target site is more than $3.5 \mathrm{~m}$ - this is the value of the (maximum) displacement response observed in the FST or the corresponding numerical simulation at the designated capacity of $150 \mathrm{~kJ}$ (Dhakal et al., 2011a).

The structure actually developed higher stresses than it should have after aided with the dissipators. For example, a numerical simulation when conducted at the same loading situation of $150 \mathrm{~kJ}$, but hitting the structure hypothetically without dissipators, revealed that the maximum cable tension in both the cases come out almost similar. The comparison is shown in Fig. 11. Thus, it became clear that the system's energy dissipation performance should be enhanced to justify the use of so many UFDs. This enhancement shall either reduce the stresses in the system, such as cable tension, at the specified impact energy of $150 \mathrm{~kJ}$, or shall increase the impact energy resistant capacity with the current state of internal forces developed in the structure. Both ways shall lead to the economic design. However, it is always beneficial if we could develop a higher energy designation (classification) of the protective structure without much difference in cost.

Where could have been the flaw in the existing system then? Towards finding a satisfactory answer, we conducted various numerical parametric simulations; basically by varying the constitutive parameters of the brake device.

\subsection{Flaw investigation}

A simulation was carried out using the constitutive parameters that correspond with the FWT behaviour; i.e. $(k)=0.0001$, and $\left(T_{0}\right)=50 \mathrm{kN}$. Figure 12 presents the corresponding cable-tension and slip-displacement time histories, compared with the behaviour in the FST. The respective time histories of the UFDs' internal and the corresponding energy dissipation efficiencies may be read out from Fig. 13. The results reveal that with the FWT-like behaviour of the UFDs, the tension in the cable is reduced appreciably and the corresponding slip-displacement is also encouraging - resulting in the energy dissipation efficiency of approximately $35 \%$ and which is more than the double of what was achieved in the FST. This reveals that the R-LPR could have been safely designated with a higher impact energy resistant capacity even if the UFDs integrated into the structure in the FST could have performed in the way they had in the FWT.

Actually the brake devices were installed into the structure manually, and we suspect that the weak performance of the UFDs in the R-LPR was due to some unknowingly introduced errors or the variations changing the behaviour of the friction-based brake devices. The potential flaw could be associated with factors such as wet surfaces involved, surface imperfection, heating up during braking, steady change between the stick- and slip-friction, errors in design, imperfections in the manufacturing process and/or the imperfections in handling of the devices during installation. However, the FWT report (Besafe, 2006) reveals a very precise design and manufacturing of the UFD devices with the precision of $\pm 5 \mathrm{kN}$ and $\pm 5 \mathrm{~cm}$, respectively, in the designated slip-tension of $50 \mathrm{kN}$ and the measured slip-displacement of $50 \mathrm{~cm}$. The FWT is a dynamic test. Moreover, the mentioned numerical results when the R-LPR is simulated with a loading situation equivalent to the FST but integrating it with the UFDs having the constitutive behaviour as demonstrated in the FWT, has already revealed a good performance with the computed energy dissipation efficiency of about $35 \%$. As for the other mentioned potential reason, no visual evidence is there to support any mishandling of the devices, 

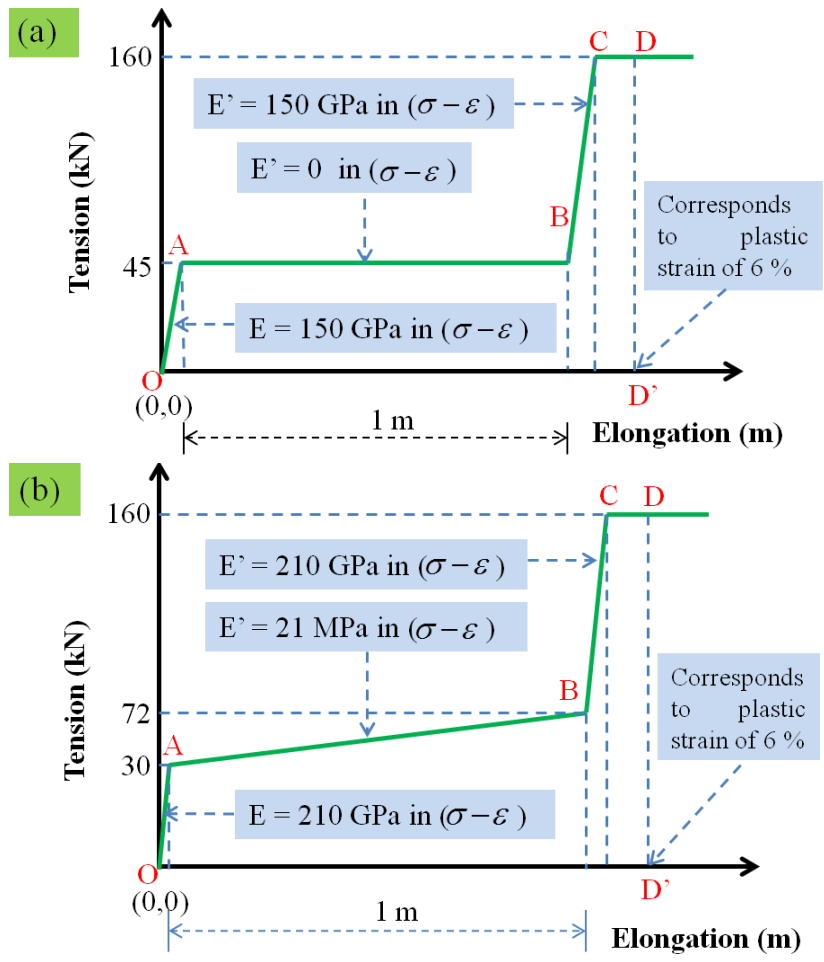

Fig. 17. Newly proposed constitutive models of the brake devices to be integrated into the R-LPR structure. (a) The Cazzani-LPR Brake Model. (b) The Dhakal-LPR Brake Model.

surface imperfections, etc. during installation. The rate dependent effects that could also be questioned here might not have been significant. This is because the velocity of slip, as obtained from the differentiation of the slip-displacement response obtained from the numerical simulation (see Fig. 14), is quite higher than the limiting range of the relative velocity between the friction surfaces, such as $0.04 \mathrm{~m} \mathrm{~s}^{-1}$, only below which it is assumed that the coefficient of dynamic friction varies significantly, i.e. from 0.2 to even greater than 0.5 (see Behrendt et al., 2011; also see David et al., 2010 for yet microscopic-level discussion on the evolution of frictional strength). Thus, the frictional rate-dependence is assumed negligible in the engineering scale.

In addition to above evidences, it is our opinion that in lieu of the production design and manufacturing and other discussed reasons, the errors should have been introduced invisibly and probably unknowingly while installing the UFDs into the R-LPR during the FST; for example, while working with the screws or bolts, ultimately due to the fact that the friction based devices are susceptible to change their performance erratically for minor changes. For instance, a critical observation of the instrumented time history of the cable tension in the FST shown in Fig. 15 seems to reveal that, there is a sudden discontinuity in the history at some value appreciably higher than $50 \mathrm{kN}$ (the designated sliptension), which should more or less correspond with the slip-

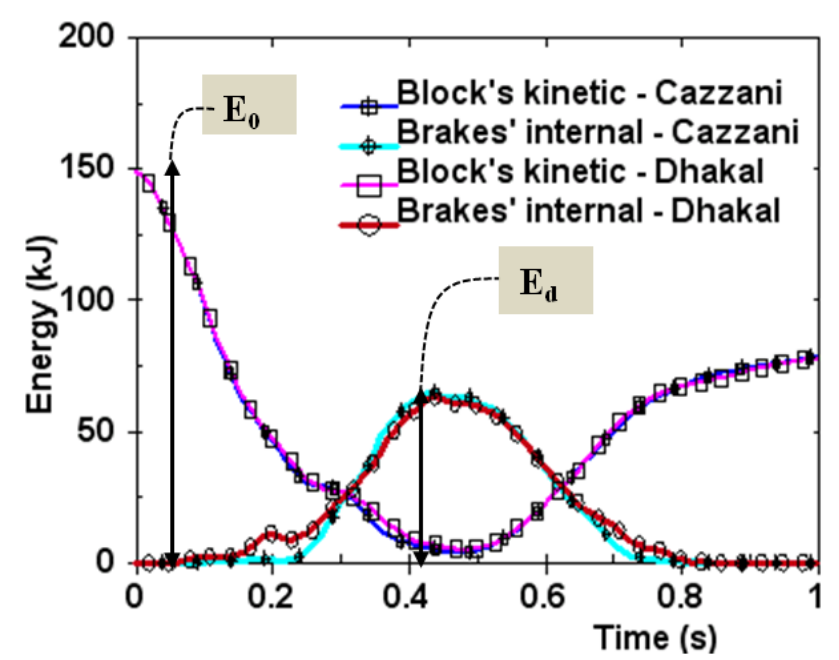

Fig. 18. Numerically simulated time histories of energy dissipation by the proposed Cazzani-LPR Brake Model and Dhakal-LPR Brake Model devices integrated separately into the R-LPR, when subjected to the designated impact energy of $150 \mathrm{~kJ}$. The energy dissipating efficiency $\left(\mathrm{E}_{d} / \mathrm{E}_{0}\right)$ of the brakes is about $43.36 \%$ in the case of the Cazzani-LPR Brake Model and $42.36 \%$ in the case of the Dhakal-LPR Brake Model. The maximum (out-of-plane) displacement response in each case was read approximately the same, about $3.9 \mathrm{~m}$.

impending stage. Therefore, it appears to disclose that the slip might have occurred at a value higher than that specified based on a series of tests in the FWT campaign. For example, the device should have been screwed or tightened with more than the predefined normal force; thereby increasing the frictional force across the groove in the U-head (also see Giacomini et al., 2008). In fact, a higher value of slip-tension results in smaller slip-displacement and larger tension, and overall a reduced energy dissipation efficiency of the brake devices. Another set of parametric analyses was conducted to confirm this, which is shown in Fig. 16.

\subsection{New proposals}

The parametric analysis with the constitutive parametric set of $T_{0}=30 \mathrm{kN}$, and $k=0.0001$ of the UFD-like brakes as shown in Fig. 16 gives an exciting energy dissipation efficiency as high as $50 \%$, but we confirmed that it couldn't utilize the cable's high tensile strength capacity overall. Significantly, from practical consideration, the LPR structures are typically meant to be installed in narrow set-back sites. It means, with the existing design of the UFDs, not only do we have a lesser energy capacity but the structure may impose greater risk by "collapsing" onto the moving road traffic to be protected due to the continuously slipping cables from the UFD brake devices; in case it has to restrain an unprecedentedly and excessively peak rockfall impact. The event may be called as the "maximum credible rockfall impact action". 
It is because the whole system, which may remain mechanically safe on its own, has a tendency to get slipped outward from the anchor supports. Thus, there should be some provision that we allow the braking systems to yield elastoplastically in extreme events. Additionally, the enhancement of energy dissipation efficiency is always desirable.

Following the above learning, two new and interesting idealized brake constitutive models were proposed for the integration into the studied structure. We named the first as "Cazzani-LPR Brake Model" to mean the brake model presented by Cazzani et al. (2002) when used in the LPR system and the second as "Dhakal-LPR Brake Model". Figure 17 shows the models, and Fig. 18 portrays the respective energy dissipation histories. Also, the time histories of the developed maximum cable tensions and the corresponding slipdisplacements are given in Fig. 19. The drop in the history of energy absorption by the UFDs after attaining the peak is due to defining the step-wise nonlinear brake model through the Cable Discrete Beam element available in LS-DYNA, which we were more confident with in modelling. If the model could be defined through a truss element formulation, then the profile of the evolution of energy absorption by the UFDs would be similar to those presented in previous figures. However, since we are interested in evaluating the energy dissipation efficiency and up to the maximum displacement stage (at around $0.4 \mathrm{~s}$ ), the modelling is acceptable. In LS-DYNA, the "*Define Curve" (LSTC, 2007) was used to define the step-wise nonlinear strain-stress data of the proposed constitutive models and the "Load Curve ID (LCID)" was used to assign the defined models to the tension-only (Cable Discrete Beam) finite elements that modelled the UFDs equivalently. As mentioned somewhere earlier, the numerically modelled UFDs had the length of $0.5 \mathrm{~m}$ and cross sectional area of $1000 \mathrm{~mm}^{2}$. The forces and elongations were converted into the respective stresses and strains accordingly.

Separate analyses of the same R-LPR system integrated with the proposed brake models at a higher impact energy ( $400 \mathrm{~kJ}$, for instance) resulted in the energy transformation time history as shown in Fig. 20 with other observed results summarized in its caption. The parametric study also reveals that the proportion of the impact energy absorbed by the UFDs does not change much with the changing impact energy level. This complies with one of the important findings by Tajima et al. (2009). As expected, the stresses in the purposefully made high strength wire-net are still safer. For example, the von-Mises stresses (shown in Fig. 21) are well below the tested breaking stress-limit of more than $1000 \mathrm{MPa}$ (see Dhakal et al., 2011a). It is, however, noteworthy that in practical situations a much irregular and very pointed block may even impact the net and the large capacity reserve may be fully mobilized or even exceeded. Our numerical analyses have not considered such a situation. Moreover, the maximum cable-tension only up to $66.44 \mathrm{kN}$ and the slipdisplacement as high as over $800 \mathrm{~mm}$ imply that, not only the energy dissipation efficiencies of the brakes have been
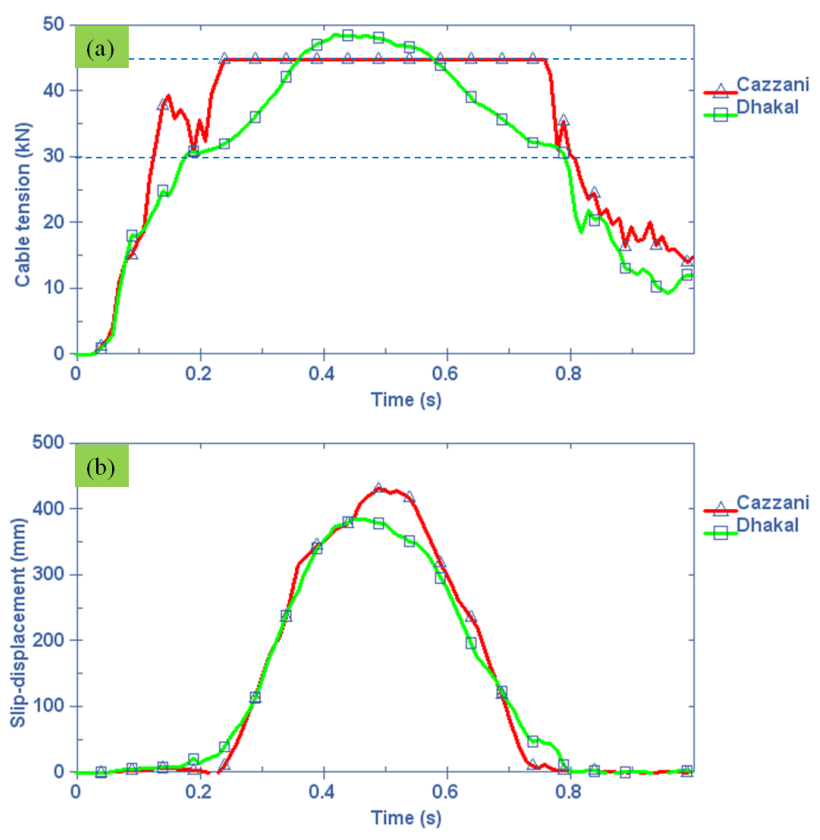

Fig. 19. Numerically simulated time histories of maximum cable tension (a) and corresponding maximum slip-displacements (at location $M$ in Fig. 1) (b) in the R-LPR installed with the UFDs having the Cazzani-LPR Brake Model and the Dhakal-LPR Brake Model respectively as the constitutive models, and subject to impact energy of $150 \mathrm{~kJ}$. The higher value of maximum tension in the cable in the Dhakal-LPR Brake Model case implies that it better utilizes the capacity of cable than the Cazzani-LPR Brake Model.

enhanced, but also the stresses in the cables have been curtailed and there is still a large reserve of the resisting capacity of the cables left to be utilized. Overall, the cable capacity is utilized more effectively in the Dhakal-LPR Brake Model case compared to the Cazzani-LPR Brake Model.

The research has thus encouragingly revealed the scope of further improvement in the design itself of the existing UFD devices being utilized in the existing LPR structures. In fact, the proposed constitutive behaviours (models) may be made practically possible. For example, the Cazzani-LPR Brake Model is possible if the additional left slip-length of the cable is made very smooth or of slightly lower diameter with a stopper at the end. Similarly, (i) maintaining the performance of the currently available UFD device (as already tested successfully by the dynamic tests) while integrating it into the structure, by minimizing the potential errors discussed earlier, (ii) and introducing a rigid stopper at the end of the freely left slip-length of the cable after a specified length (say $1 \mathrm{~m}$ ) and (iii) changing the designated slip-tension to $30 \mathrm{kN}$ preferably during manufacturing, as shown schematically in Fig. 22, the Dhakal-LPR Brake Model may be materialized. The use of the stopper allows the system to undergo even the elasto-plastic deformation after brake (along the path B-C-D shown in the models) in the unprecedented 


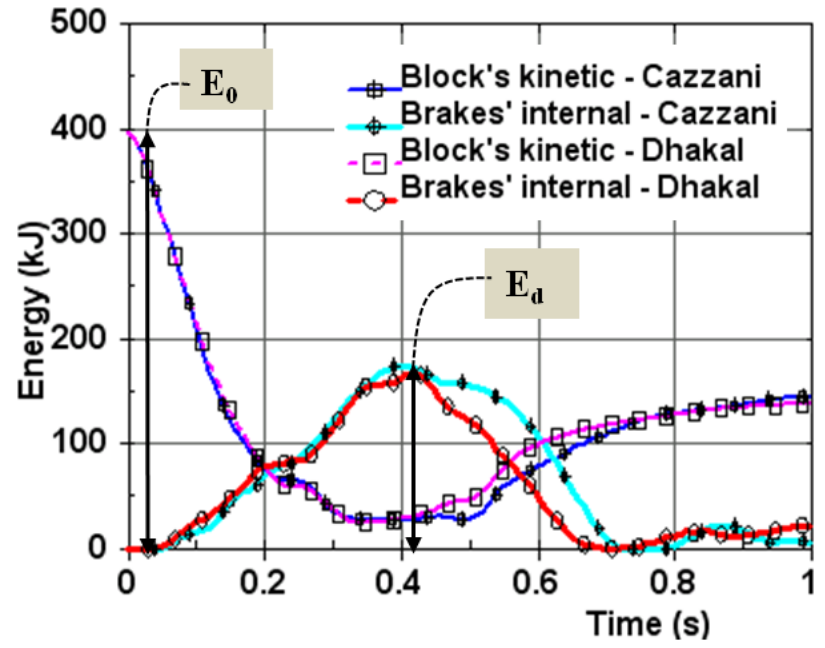

Fig. 20. Numerically simulated time histories of energy dissipation by the brake devices having the proposed consitutive models defined by Cazzani-LPR Brake Model and Dhakal-LPR Brake Model and integrated separately into the R-LPR, when subjected to the impact energy of $400 \mathrm{~kJ}$ (2.67 times higher than the originally designated system capacity of $150 \mathrm{~kJ}$ ). The maximum (out-of-plane) displacement response in each case was read approximately the same, about $4.9 \mathrm{~m}$. Correspondingly the maximum tension in the cable and the maximum slip-displacement (again at location $M$ in Fig. 1) were found, respectively, to be $45 \mathrm{kN}$ and $847 \mathrm{~mm}$ in the case of the Cazzani model while $66.44 \mathrm{kN}$ and $767 \mathrm{~mm}$ in the case of the Dhakal model.

extreme loading (say, the most credible rockfall impact action); thereby not allowing the mechanically safe structure to fall onto the seemingly protected possible road traffic very close to the intercepting structure. Thus, the proposals appear to have certain implications also for the performance enhancement in terms of the control of displacement in addition to the major objective of aiding for the higher resistant capacity of the structure.

\section{Conclusions and recommendations}

A combined numerical and analytical investigation was conducted towards the anticipated performance enhancement of a particular type of rockfall protective cable-net structure (barrier) known as Long-span Pocket-type Rock-net (LPR), developed newly in Japan. The LPR configuration of $15 \mathrm{~m}$ span and the $150 \mathrm{~kJ}$ designated capacity, which was successfully modelled numerically as well as analytically in the companion paper Dhakal et al. (2011a) was taken as the reference structure, referred to as R-LPR.

The research started following one of the important conclusions of the previous work that: owing to the safer stress states in the prevailing design and to the typical target application (i.e. just beside the road carriageway with a limited

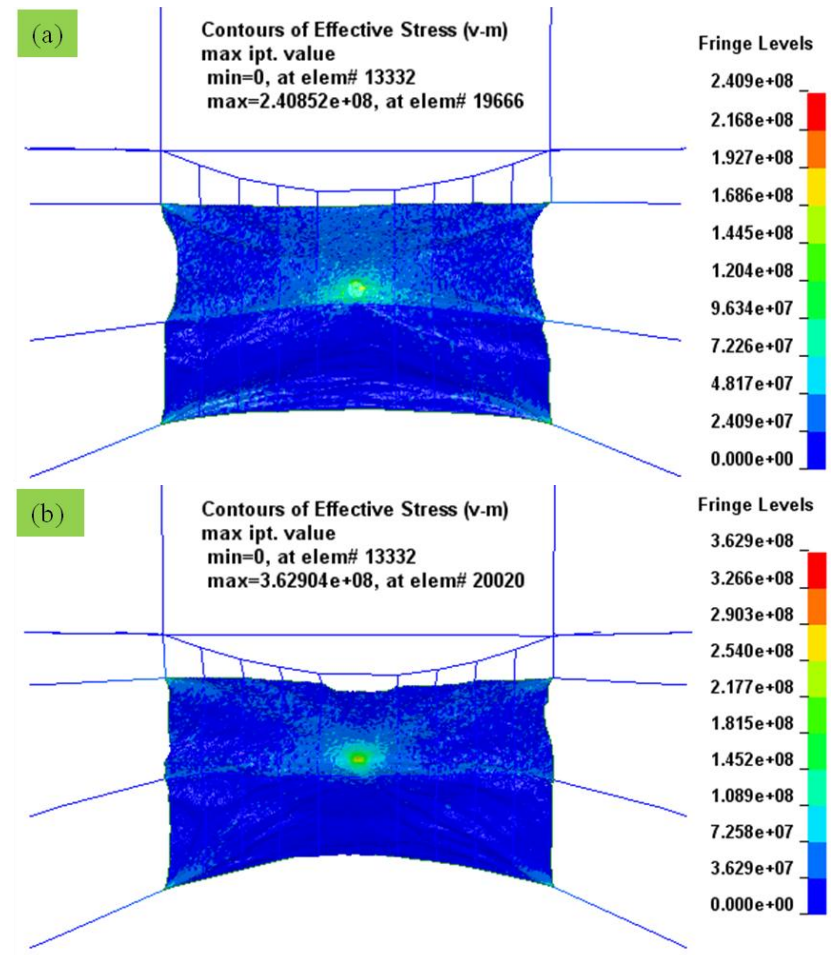

Fig. 21. Numerically simulated von-Mises stress distribution in the R-LPR structure integrated with the Dhakal-LPR Brake Model devices subject to rockfall impact energies of $150 \mathrm{~kJ}$ (a) and $400 \mathrm{~kJ}$ (b) hit by the idealized cylindrical block equivalent to that used in the past full-scale modelling.

space in between), the displacement response (i.e. the outof-plane displacement of wire-net) is possibly the most vital performance-evaluating response-quantity for the LPR structures in general, as long as the barrier components are strong enough. The criterion is based on the fact that the structure should not pose risks onto the road-traffic while it restrains the falling rock-boulder albeit the structure itself is mechanically safe.

Towards identifying solutions for controlling this displacement response, a manipulation in the structural mass, for example, as suggested by the displacement-predictive correlation, was endeavoured and various correlations have been disclosed. Accordingly, the wire-net's mass based parametric computations were carried out numerically. In particular, a clear picture of achieving the control of displacement in the LPR structure through the increment in the mass of the wire-net in terms of its density and equivalent thickness was seen. The considered parameters of the wire-net can be achieved by changing the spacing of the woven mesh and/or the diameter of the weaving wire in reality. In general, a closely fitting power correlation with positive index between the defined "Displacement controlling mass factor" $\left(F_{\mathrm{M}}\right)$ and "Mass increment factor" (I) was obtained. Use of hybrid mesh (such as a double layer mesh) was also identified as an 
alternative solution to the control of displacement based on the discussed mass and stiffness increments of the wire-net. However, the practicality and the quantification of the effectiveness of such a solution are left for further investigation in the future.

Additionally, through analytical computations, the effects of block velocity or mass (or size) in the displacement performance of an LPR structure have been very systematically illustrated. Another closely fitting power law (now with negative exponent) between the defined "Displacement response velocity factor $\left(F_{\mathrm{v}}\right)$ " and "Impact velocity $\left(\mathrm{V}_{0}\right)$ " was seen. An analysis also tended to reveal that due to the typical features of the LPR design consisting of very strong wire-net and small sized chain-mesh, meant to be installed in the described narrow space between the hill-slope and the roadcarriageway (thereby demanding a controlled displacement), these systems may be especially recommended in some selected sites where the LPR structure is subjected to smaller size, higher velocity type rockfall impacts. In particular, Japan most often witnesses such a scenario, as inferred from a past study. Furthermore, a prospect of using the LPR structures even in the wider-set-back sites subjected to higher velocity impacts was also introduced.

The second aspect of performance enhancement was smaller increasing the energy dissipation efficiency of the brake devices, the capacity designation of the whole structure. In the previous study on LPR structures (including the companion paper), the primary concern was the overall verification of the newly developed structural system. There was no detailed evaluation of the brake devices, i.e. the UFDs. However, in this paper, through the observation of the numerically simulated energy transformation time history response of the studied R-LPR, it was identified that the UFDs integrated into the structure really could not perform satisfactorily in the FST.

Following a rigorous analysis as discussed in Sect. 3.2, we reached a conclusion that: in lieu of the production design and manufacturing, and other discussed factors, the errors should have been introduced invisibly and probably unknowingly while installing UFDs into the R-LPR during the FST, for example, while working with the screws or bolts; ultimately due to the fact that the friction based devices are susceptible to change their performance erratically for minor changes. The measured time history in the FST also tended to witness a higher value of slip-tension mobilized. Additionally, a subsequent numerical parametric study suggested that the energy dissipation efficiency of the UFDs reduces significantly with the increase in slip-tension $(\mathrm{kN})$, approximately at the rate of 1:2.

Therefore, in general, a very careful handling of UFDlike devices is importantly recommended. Utmost quality control should be assured, for example, while tightening the screws or bolts during installation which are likely to affect the friction-brake devices properties such as the slip-tension limit. In addition to all, further investigation based on micro-

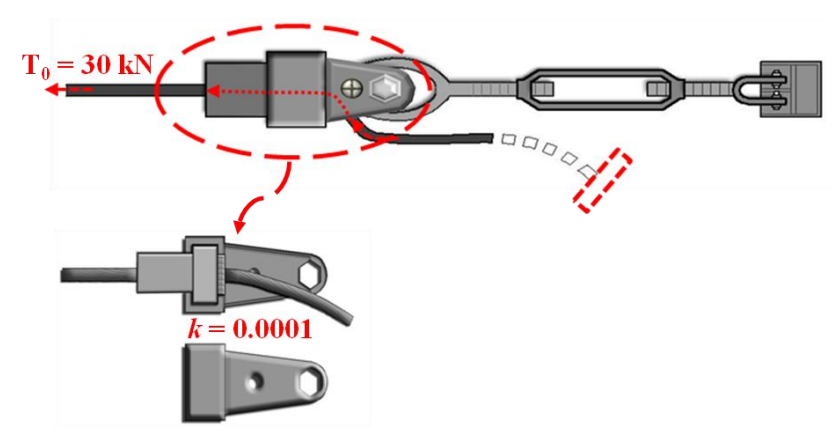

Fig. 22. Schematic drawing for the physical impression of the potential Dhakal-LPR Brake Model device. Note the stopper.

modelling of slip-stick (e.g. Diaz et al., 2010) of the UFDtype friction brakes proposed to be effectively utilized in the LPR-like rockfall protective structures is also encouraged.

Furthermore, the parametric investigations not only identified the potential flaw and recommended accordingly to achieve the FWT-equivalent role of the UFDs also when integrated into the LPR structure, but also suggested the prospects of even modification/improvement in their existing design. It is both for the increased energy dissipation as well as to mitigate the potential risk of the falling rock restraining structure hitting the moving road traffic, for instance, in the case of an extreme loading, say, the "maximum credible rockfall impact action". Following this conclusion, two innovative constitutive models, namely the "CazzaniLPR Brake Model" and the "Dhakal-LPR Brake Model" (see Fig. 17), were proposed which could dissipate a large proportion (over $40 \%$ ) of the impact energy to be resisted by the structure by "yielding" a larger slip-displacement, but still limiting the internal forces in the structure well within safe limits. The latter feels more effective and practical in the sense that (a) the inclined plateau of hardening has been practically observed and already determined based on the element-level dynamic tests (FWT) of the friction behaviour of such devices, and (b) they develop some force in the cable even after slip-tension, and hence utilize the cable's capacity in moderate-level impacts also. This particular brake device may be achieved by introducing a stopper-like arrangement at the end of the predetermined slip-length of the existing UFD device with controlled frictional properties (see Fig. 22).

Therefore, we finally conclude that the existing capacitydesignations of the LPR structures have encouraging scopes of performance enhancement by effectively controlling the out-of-plane displacement, and upgrading the system's capacity by many-fold even only through an improved brake technology, keeping the same original configuration and composition of the cable-net component.

Acknowledgements. The authors thank Daiichi Consultants Co., Kochi, Japan (particularly the chairperson, T. Ushiro), Nihon Protect Co., Matsuyama, Japan and Besafe Consultants Co., 
Niigata, Japan for the necessary test results used in the research. The first author also acknowledges N. T. K. Lam, The University of Melbourne, Australia, and A. Volkwein, WSL, Switzerland, for their kind e-mail responses, comments and helpful suggestions at times. The authors are especially thankful to the two anonymous referees whose very high quality reviews with gateway questions and suggestions changed the whole face of the paper and brought it to the present standard. Finally, the first author is thankful to his wife S. Acharya (Dhakal) for her continuous support and D. Neupane for his participation in some of the important discussions.

Edited by: T. Glade

Reviewed by: two anonymous referees

\section{References}

Ali, M., Lam, N., Zhang, L., and Gad, E.: Impact resistant behaviour of lightweight structural elements, International Journal of Aerospace and Lightweight Structures, 1, 179-201, 2011.

Behrendt, J., Weiss, C. , and Hoffman, N. P.: A numerical study on stick-slip motion of a brake pad in steady sliding, J. Sound. Vib., 330, 636-651, 2011.

Besafe: E-fence experimental report. Besafe Co. Ltd., Niigata, Japan, 2006 (in Japanese).

Cantarelli, G., Giani, G. P., Gottardi, G., and Govoni, L.: Modelling rockfall protection fences, Web Proc. Of First World Landslide Forum, Tokyo, Japan, 662-667, 2008.

Cazzani, A., Mongiovi, L., and Frenez, T.: Dynamic finite element analysis of interceptive devices for falling rocks, Int. J. Rock. Mech. Min. Sci., 39, 303-321, 2002.

David, O. B., Rubinstein, S. M., and Fineberg, J. : Slip-stick and the evolution of frictional strength, Nature, 463, 76-79, 2010.

Diaz, J. J. d. C., Nieto, P. J. G., Castro-Fresno, D., and Hernandez, J. R.: Nonlinear explicit analysis and the study of the behaviour of new ring-type brake dissipator by FEM and experimental comparison, Appl. Math. Comput., 216, 1571-1582, 2010.

Dhakal, S., Bhandary, N. P., Yatabe, R., and Kinoshita, N.: Experimental, numerical and analytical modelling of a newly developed rockfall protective cable-net structure, Nat. Hazards Earth Syst. Sci., 11, 3197-3212, doi:10.5194/nhess-11-3197-2011, $2011 \mathrm{a}$.

Dhakal, S., Bhandary, N. P., Yatabe, R., and Kinoshita, N.: A proposal on improved impact load specification for the design of a rockfall interceptive cable-net structure, Proc. of 9th International Conference on Shock and Impact Loads on Structures, Fukuoka, Japan, 269-278, 16-18 November 2011b.

Dhakal, S., Bhandary, N. P., Yatabe, R., and Kinoshita, N.: Performance evaluation of an energy dissipator in a rockfall protective cable-net structure, Proc. of the 13th International Summer Symposium of Japan Society of Civil Engineers, Kyoto, 43-46, 2011c.

Dhakal, S., Bhandary, N. P., Yatabe, R., and Kinoshita, N.: Towards characterization of rockfall impact loading onto a protective cable-net barrier, submitted, Rock Mech. Rock Eng., 2012.
Giacomini, A., Giani, G. P., and Migliazza, M.: Quasi-static and dynamic response of energy dissipators for rockfall protection, Proc. of 1st Southern Hemisphere International Rock Mechanics Symposium (SHIRMS 2008), Perth, Western Australia, 10 pp., 2008.

Kinoshita, N.: Development of long-span pocket-type rock-net, Proc. of Annual Meeting of Ministry of Land, Infrastructure, Transport and Tourism, Shikoku Regional Development Bureau, Japan, 4 pp., 2009 (in Japanese).

Lam, N. T. K., Tsang, H. H., and Gad, E. F.: Simulations of response to low velocity impact by spreadsheet, Int. J. Struct. Stab. Dy., 10, 1-17, 2010.

LSTC: LS-DYNA Keyword User's Manual, Version 971, I-II, Livermore Software Technology Corporation (LSTC), USA, 2206 pp., 2007.

Matsuo, O., Tsutsumi, T., and Sasaki, T.: Current states of practices and technical issues of rockfall disasters and their mitigation measures in Japan, in: Proc. of the Joint Japan-Swiss Scientific Seminar on Impact Load by Rock Falls and Design of Protection Structures, edited by: Masuya, H. and Labiouse, V., Kanazawa, Japan, 7-12, 1999.

Peila, D. and Ronco, C.: Technical Note: Design of rockfall net fences and the new ETAG 027 European guideline, Nat. Hazards Earth Syst. Sci., 9, 1291-1298, doi:10.5194/nhess-9-1291-2009, 2009.

Peila, D.: Chapter 12, Ground reinforced embankments for rockfall protection - From real scale tests to numerical modelling, in: Rockfall Engineering, edited by: Lambert S., Nicot, F., , John Wiley \& Sons, New York, ISTE Ltd., London, ISBN 9781-84821-256-5, 2011.

Tajima, T., Maegawa, K., Iwasaki, M., Shinohara, K., and Kawakami K.: Evaluation of pocket-type rock net by full-scale tests, Proc. of 33rd IABSE Symposium, Bangkok, Thailand (http://133.28.114.77/maegawa/ IABSE-Symposium2009-212_07_01.pdf), 8 pp., 2009.

Volkwein, A.: Numerical simulation of flexible rockfall protection systems, Diss. ETH, Nr. 15641, PhD thesis, ETH, Zurich (http://e-collection.library.ethz.ch/eserv/eth:27491/ eth-27491-02.pdf), 134 pp., 2004 (in German).

Volkwein, A., Melis, L., Haller, B., and Pfeifer, R.: Protection from landslides and high speed rockfall events: Reconstruction of Chapman's Peak Drive, Proc. of IABSE Symposium, Structures and Extreme Events, Lissabon, Portugal, 47-54, 2005.

Volkwein, A., Roth, A., Gerber, W., and Vogel, A.: Flexible rockfall barriers subjected to extreme loads, Structural Engineering International, 19, 327-332, 2009.

Yang, Y., Lam, N. T. K., and Zhang, L.: Evaluation of simplified methods of estimating beam responses to impact, Int. J. Struct. Stab. Dy., accepted, 20 pp., on 5 March 2011.

Yoshida, H.: Recent experimental studies on rockfall control in Japan, in: Proc. of the Joint Japan-Swiss Scientific Seminar on Impact Load by Rock Falls and Design of Protection Structures, edited by: Masuya, H. and Labiouse, V., Kanazawa, Japan, 69 $78,1999$. 